\title{
Genetic identification of C-fibers that detect massage-like stroking of hairy skin in vivo
}

\author{
Sophia Vrontou ${ }^{1}$, Allan M. Wong ${ }^{1,2}$, Kristofer K. Rau ${ }^{3}$, H. Richard Koerber ${ }^{4}$, and David J. \\ Anderson ${ }^{1,2}$ \\ ${ }^{1}$ Division of Biology 156-29, California Institute of Technology, Pasadena, California 91125, USA \\ ${ }^{2}$ Howard Hughes Medical Institute, California Institute of Technology, Pasadena, California \\ 91125, USA \\ ${ }^{3}$ Department of Anatomical Sciences and Neurobiology, University of Louisville, Louisville, KY \\ 40202-1702 \\ ${ }^{4}$ Department of Neurobiology, University of Pittsburgh, Pittsburgh, Pennsylvania 15261, USA
}

\section{Abstract}

Stroking of the skin produces pleasant sensations that can occur during social interactions with conspecifics, such as grooming ${ }^{1}$. Despite numerous physiological studies (reviewed in ref. ${ }^{2}$ ), molecularly defined sensory neurons that detect pleasant stroking of hairy skin ${ }^{3,4}$ in vivo have not been reported. Previously, we identified a rare population of unmyelinated sensory neurons that express the G protein-coupled receptor (GPCR) MrgprB4 $4^{5,6}$. These neurons exclusively innervate hairy skin with large terminal arborizations ${ }^{7}$ that resemble the receptive fields of C-tactile (CT) afferents in humans ${ }^{8}$. Unlike other molecularly defined mechanosensory C-fiber subtypes ${ }^{9,10}$, MrgprB $4^{+}$neurons could not be detectably activated by sensory stimulation of the skin ex vivo. Therefore, we developed a preparation for calcium imaging in their spinal projections during stimulation of the periphery in intact animals. MrgprB4 ${ }^{+}$neurons were activated by massage-like stroking of hairy skin, but not by noxious punctate mechanical stimulation. By contrast, a different population of C-fibers expressing MrgprD ${ }^{11}$ was activated by pinching but not by stroking, consistent with previous physiological and behavioral data ${ }^{10,12}$. Pharmacogenetic activation of MrgprB4- expressing neurons in freely behaving animals promoted conditioned place preference ${ }^{13}$, suggesting that such activation is positively reinforcing and/or anxiolytic. These data open the way to understanding the function of MrgprB4 neurons during natural behaviors, and provide a general approach to functionally characterizing genetically identified subsets of somatosensory neurons in vivo.

In isolated skin-nerve preparations, MRGPRB4+ neurons were not electrophysiologically activated by mechanical, thermal or chemical stimuli (see Supplementary Note 1). Therefore, we sought to perform calcium imaging specifically in these neurons while stimulating the periphery of intact mice. To target genetically encoded calcium sensors to $\mathrm{MRGPRB}^{+}$or MRGPRD ${ }^{+}$neurons, we injected neonatal Mrgprb4- $t d T$ Tomato-2A-cre mice (Supplementary Fig. 1) or MrgprD-EGFP-cre mice ${ }^{10}$ intraperitoneally (i.p.) with a Credependent adeno-associated virus (AAV) expressing GCaMP3.0 (ref. 14) (Supplementary Table 1, Methods and Supplementary Note 2). A similar efficiency of viral expression (62 \pm

Correspondence and requests for materials should be directed to D.J.A. (wuwei@ caltech.edu)..

Author contributions: S.V. carried out all imaging and behavioral experiments, A.W. helped configure the 2-photon imaging system and developed the light grid method, K.R. and H.R.K. carried out electrophysiological recordings in isolated skin-nerve preparations,

D.J.A. participated in experimental design and data interpretation and wrote the manuscript together with S.V. 
3.6\%) was observed in MrgprD-EGFP-cre mice (Fig. 1b, Supplementary Fig. 2a-c, g and Supplementary Note 2). This approach yielded relatively efficient expression of the genetically encoded calcium sensor in MRGPRB4::tdTomato ${ }^{+}$dorsal root ganglia (DRGs) neurons $(62+6 \%)$ along the rostro-caudal axis in adult mice (Fig. 1a, c, Supplementary Fig. 2d-f, h and Supplementary Note 2). Expression of GCaMP3.0 or mGCaMP3.0 was especially robust in the central spinal projections of these neurons (Fig. 1d, e). No expression of the reporter was observed in virally injected wild-type mice.

To record calcium transients in the central projections of $\mathrm{MrgprD}^{+}$or $\mathrm{B}^{+}$neurons, we performed two-photon imaging through a spinal cord laminectomy while stimulating the intact animal (Supplementary Note 3). We first tested responses to centrally or peripherally applied chemical stimuli. Direct application to the spinal cord of depolarizing concentrations of $\mathrm{KCl}$ elicited robust increases in $\Delta \mathrm{F} / \mathrm{F}$ in both $\mathrm{MrgprD}^{+}$fibers (Fig. 1g, i, m; Mean Percent Increase in peak $\Delta \mathrm{F} / \mathrm{F}\left(\mathrm{MPI}[\Delta \mathrm{F} / \mathrm{F}]_{\text {peak }}\right)=222 \pm 19 \%( \pm \mathrm{SEM}) ;$ Mean Latency to Peak (MLP) $=8.6 \pm 3.6 \mathrm{sec}, \mathrm{n}=3$ ) and MrgprB4${ }^{+}$fibers (Fig. 1h, j, n; MPI $[\Delta \mathrm{F} / \mathrm{F}]_{\text {peak }}=201.6 \pm 33.2 \%$, MLP $=9.3 \pm 4.15 \mathrm{sec}, \mathrm{n}=3)$. We also observed responses to $a, \beta$ methylene $(\mathrm{Me})$ ATP, a ligand known to activate both $\mathrm{MrgprD}^{+}$and MrgprB4${ }^{+}$neurons in vitro ${ }^{7,15}$, via both direct spinal application and/or peripheral injection into glabrous or hairy skin of the hindpaw, respectively (Fig. 1k-n and Supplementary Fig. 3; see also Supplementary Note 4). Finally, MrgprB4 ${ }^{+}$central fibers were activated by peripheral injection of capsaicin in mice genetically engineered to express $\mathrm{TrpV} 1$ in MrgprB4 ${ }^{+}$neurons, which normally do not express this channel ${ }^{7}$ (Supplementary Fig. 4 and Note 4). Thus our preparation was able to detect calcium transients in both $\mathrm{MrgprD}^{+}$and $\mathrm{MrgprB}^{+}$fibers by peripheral injection of specific chemical stimuli that activate these neurons.

We next tested whether this preparation could be used to image activity evoked by mechanical stimulation of the periphery. We first measured activity in MRGPRD ${ }^{+}$fibres after mechanical stimulation of the hindpaw using a custom pinching device (see Methods) (Fig. 2a). In agreement with their established role in sensing noxious punctate mechanical stimuli $^{10,12}$, MRGPRD $^{+}$fibres were strongly activated by trains of pinching stimuli in the ipsilateral hindpaw (and more specifically in the particular experiment by pinching of the most distal ipsilateral digit) (Fig. $2 \mathrm{~d}$, e; $\mathrm{MPI} \Delta \mathrm{F} / \mathrm{F}_{\text {peak }}=77.8+8.9 \%, \mathrm{n}=7$ trials per mouse). Responses were restricted to a subset of GCaMP3.0-expressing fibres within a given imaging field, whereas other fibres were unresponsive (Fig. 2c-e, h and Supplementary Fig. 5a-e). This heterogeneity probably reflects the different receptive fields of these fibres relative to the site of stimulation. Responses of $\mathrm{MRGPRD}^{+}$fibres to pinching were reproducible across trials and mice (Fig. 2d, e, h, j and Supplementary Table 2), and also specific to the ipsilateral hindpaw and to particular digits (Supplementary Fig. 6 and Supplementary Note 5).

Importantly MrgprD ${ }^{+}$fibers in a given ROI that were activated by pinching were not activated when the last digit of the ipsilateral hindpaw was stroked lightly using a brush (Fig. 2f-g, i). The same fibers could, however, be reactivated by a subsequent pinching stimulus (Supplementary Fig. 9i-1), indicating that the lack of response to brushing was not due to adaptation or desensitization produced by the pinch stimulus. These data therefore suggest a specificity of $\mathrm{MrgprD}^{+}$fibers for punctate or focal noxious mechanical stimulation of the skin, consistent with previous physiological and behavioral studies of this subpopulation $^{10,12}$.

To identify mechanical stimuli that activate MrgprB4+ fibers, we tested a variety of innocuous mechanical stimuli designed to simulate natural stroking or grooming, using a custom-designed brush (Fig. 3a, Supplementary Fig. 14 and Methods). Calcium transients in MrgprB4-tdTomato ${ }^{+}$fibers (Supplementary Fig. 7) were elicited by repeated stroking 
$(0.2-0.5 \mathrm{~Hz})$ of relatively large areas $(2-3 \mathrm{~mm} \times 20-30 \mathrm{~mm})$ of posterior dorsal thoracic and proximal hindlimb hairy skin (Fig. 3c-e, h; green traces)), consistent with the distribution of $\mathrm{MrgprB}^{+}{ }^{+}$fibers in the periphery ${ }^{7}$. The average forces and velocities delivered from these manual stimuli, which included a mild pressure component, were relatively dynamic but fell within the range of $20-90 \mathrm{mN}$ and a speed of $0.5-2 \mathrm{~cm} / \mathrm{sec}$ (see Supplementary Methods). As in the case of $\mathrm{MrgprD}^{+}$neuron responses to pinching, responses in $\mathrm{MrgprB}^{+}$neurons to stroking were observed in particular fibers in a given field of view, were specific to the ipsilateral side of the animal and particular areas of the skin, and were reproducible across trials and animals (Fig. 3, Supplementary Figs. 5, 7 and 8, Supplementary Table 3 and Supplementary Note 6). In contrast to $\mathrm{MrgprD}^{+}$fibers, MrgprB4 ${ }^{+}$fibers were not activated by localized pinching of hairy skin in regions activated by stroking (Fig. 3f-g, i), and this selectivity was not due to desensitization (Supplementary Fig. 9a-f). These data indicate that $\mathrm{MrgprB}^{+}$fibers are activated by massage-like stroking of hairy skin. Thus, the two classes of cutaneous C-fibers marked by expression of MrgprB4 and MrgprD, respectively, respond to distinct types of mechanical stimulation in vivo. The reason why MrgprB4 $4^{+}$fibers are not also activated by pinching is not clear, but could reflect their specific tuning to moving stimuli ${ }^{16}$.

The stimuli used to activate MrgprB4 $4^{+}$fibers were designed to mimic stroking and allogrooming stimuli. The social interactions associated with such stimuli have been shown to be positively reinforcing in juvenile mice ${ }^{17}$, using a conditioned place preference (CPP) assay ${ }^{13,17}$, suggesting that these stimuli may have a positive affective valence ${ }^{18}$. We therefore asked whether direct activation of $\mathrm{MrgprB}^{+}$neurons could similarly promote a preference for the location in which this stimulation occurred, using a pharmacogenetic strategy. Juvenile (1 month-old) MrgprB4-Cre male mice were injected neonatally with an AAV encoding the $\mathrm{hM} 3\left(\mathrm{G}_{\mathrm{q}}\right.$-coupled) DREADD ${ }^{19}$, whose activation by clozapine-N-oxide (CNO) causes membrane depolarization (Fig. 4a). Calcium imaging experiments confirmed that $\mathrm{CNO}$ was able to induce calcium transients in MrgprB44 ${ }^{+}$spinal afferent fibers coexpressing GCaMP3.0 and hM3DREADD (Supplementary Fig. 10 and Supplementary Note 7).

We hypothesized that activation of MrgprB4 ${ }^{+}$neurons would promote a preference for the chamber associated with CNO treatment. Since most mice during a pre-training exposure to the CPP apparatus showed an initial preference for one of the two side chambers (Fig. 4b, "I.P." and Supplementary Fig. 11) we employed a biased design ${ }^{20,21}$ to test whether activation of MrgprB4 ${ }^{+}$neurons would increase the animals' preference for the initially nonpreferred (I.N.P.) chamber. To do this, mice were conditioned over 4 days (experimenter blind to genotype) by pairing a $1 \mathrm{hr}$ exposure to $\mathrm{CNO}$ with the I.N.P. chamber on each of two days, alternating with exposure to saline in the I.P. chamber (Fig. 4c, lower).

When tested on the day after conditioning, Mrgprb4-hM3DREADD mice (Fig. 4e), but not a series of control mice (Fig. $4 \mathrm{f}-\mathrm{i}$ ), exhibited a statistically significant increase in the time they spent in the I.N.P. chamber that was paired with CNO exposure (Fig. 4c-d; 190+95\% increase, $\mathrm{P}<0.01$ pre- versus post-training, $\mathrm{n}=15$ mice; see Supplementary Fig. 12 for scatterplots; Supplementary Fig. 13f and Supplementary Note 8). The mean difference score of the experimental animals in the I.N.P. chamber (Fig. 4e, j; 253+65.8) was significantly higher than that of the pooled controls (Fig. 4j; $51.8+35.3$, $\mathrm{t}=2.92, \mathrm{P}<0.01, \mathrm{n}=33$ mice) and our statistical power $(0.83)$ was sufficient to detect this difference given the effect size (difference of means $=201+70$; 95\% confidence interval: $62.9-339.3$; Cohen's $\mathrm{d}=0.872$ ). There was no statistically significant difference between groups in the time spent in the I.N.P. chamber during the pre-test (Supplementary Fig. 12). These data suggest that artificial activation of $M R G P R B 4^{+}$neurons in Vivo is positively reinforcing and/or anxiolytic ${ }^{18,21}$. In contrast, artificial activation of $\mathrm{MRGPRD}^{+}$neurons using CNO and DREADD produced 
neither CPP (Fig. 4d, h and Supplementary Fig. 12d and 13d) nor (in separate experiments) conditioned place aversion ${ }^{22,23}$ (CPA; data not shown). The failure to obtain CPA using MRGPRD::DREADD mice may reflect technical or biological factors. For example, although hindpaw injection of formalin produces $\mathrm{CPA}^{22}$, MRGPRD ${ }^{+}$neurons are not required for nocifensive responses to formalin ${ }^{24}$

Here we report the first application of calcium imaging to record physiological responses of primary sensory neurons to cutaneous stimulation in an intact animal. Using GECIs, we identify for the first time a molecularly defined subpopulation of unmyelinated fibers that responds to innocuous stroking of hairy skin in vivo. Selective manipulation of these neurons in vivo also provides the first example of a genetically identified population of Cfibers whose functional activation has a positive ${ }^{18}$ rather than negative ${ }^{23}$ behavioral valence.

Our results provide proof-of-principle for a means to link molecular identity to stimulus selectivity for primary sensory neuron subtypes that cannot be functionally characterized using more conventional approaches. The inability to detect activation of MrgprB4 neurons by mechanical stimuli in isolated skin-nerve preparations ${ }^{10}$, for whatever reason(s) (see Supplementary Discussion 1), clearly distinguishes them functionally from other populations of unmyelinated mechanosensitive neurons that have been recently characterized in this manner ${ }^{9}$.

In humans, C-LTMRs, also called C-tactile (CT) afferents ${ }^{2}$, have been associated with gentle, pleasant stroking of hairy skin ${ }^{25}$. Although the unmyelinated axons of MrgprB4${ }^{+}$ neurons ${ }^{7}$ indicates they are C-fibers, it is not possible to classify them as low-threshold mechanoreceptors according to the electrophysiological criteria established for C-LTMRs ${ }^{26}$ since they are not activated by von Frey filaments. Nevertheless, our imaging and behavioral data taken together suggest that MrgprB4 ${ }^{+}$neurons detect massage-like stroking of hairy skin that is pleasant or rewarding, supporting the hypothesis that they may constitute at least one class of CT afferents ${ }^{7}$. Further studies will be required to determine whether sensory neurons with similar properties exist in humans. In mice, activation of these neurons may normally occur during social (affiliative or maternal) interactions, during self-stimulation or other behavioral conditions. Identification of these conditions will enable assessment of the requirement of $\mathrm{MrgprB}^{+}$neurons for specific behavioral responses, via loss-of-function experiments. The functional characterization of a novel population of CT afferents in the mouse now opens the way to identifying the molecular transduction mechanisms operating in these cells, and the higher-order circuitry that these neurons engage to produce a positive affective state ${ }^{27}$.

\section{METHODS SUMMARY}

Mice expressing reporters and/or Cre recombinase targeted to the $\mathrm{MrgprB}_{4}$ locus $^{7}$ were generated by homologous recombination in embryonic stem cells, according to standard procedures. Heterozygous neonates from MrgprD-Cre and MrgprB4-Cre mice were injected intraperitoneally ${ }^{28}$ with Cre-dependent AAV8 viruses expressing G-CaMP3.0 ${ }^{14}$ or hM3 $\left(\mathrm{G}_{\mathrm{q}^{-}}\right.$ coupled) DREADD ${ }^{19}$, and imaged as adults ( $\geq 8$ weeks old).

Electrophysiology experiments on ex vivo skin-nerve preparations from adult heterozygous MrgprB4-EGFPf reporter mice were performed as described ${ }^{26}$. For calcium imaging, following a dorsal laminectomy the spinal column was stabilized ${ }^{29,30}$ and filled with imaging solution (see Supplementary Methods). Imaging was performed using a 2-photon laser scanning microscope (Ultima, Prairie Inc) using an Olympus 40X 0.8 N.A water immersion objective, at 128x128 pixel resolution with an acquisition rate of 8-12 frames per second. Mechanical stimuli were delivered using a custom-modified No. 5 sable paint brush 
or serrated forceps, in a manner electronically time-stamped to image acquisition (Supplementary Fig. 14 and Methods). Stimuli were delivered to each mouse in a series of trials, separated by a few minutes; each trial consisted of one or more stimuli delivered typically at intervals of several seconds. Chemical stimuli were delivered to the spinal cord using a Pipetman and to the periphery using a syringe pump. Calcium responses were analyzed using custom software written in Matlab (see Methods). For calculating $\Delta F / F$ $\left[\left(\mathrm{F}_{\mathrm{av}}-\mathrm{F}_{0}\right) / \mathrm{F}_{0}\right], \mathrm{F}_{0}$ is the average of the first 10 frames of the recording period.

For behavioral experiments, juvenile (1 month-old) mice neonatally injected with Credependent AAV8 were subjected to a conditioned place preference assay $\left(\mathrm{CPP}^{13}\right)$ using a biased design ${ }^{20,21}$, by an investigator blind to genotype. All animals were tested for their initial chamber preference prior to conditioning (see Fig. 4c).

All data were analyzed for statistical significance using repeated measure ANOVAs (unless stated otherwise). Following detection of a significant interaction and/or main effect, Bonferonni-corrected post hoc comparisons of means were performed. Details of statistical analysis are available in Methods.

\section{METHODS}

\section{Animals}

Animals were grouped housed, unless otherwise mentioned, at $23{ }^{\circ} \mathrm{C}$ with ad libitum access to food and water in a 13-h day/11-h night cycle, with the day starting at 07:00. All animal procedures were performed under protocols approved by the Caltech Institutional Animal Care and Use Committee (IACUC).

\section{Generation of MrgprB4 Knock-in mice}

MrgprB4-mtdTomato-2A-NLScre-frt-PGK-neo-frt and MrgprB4-EGFPf-2A-FLP-ACN mice were generated via standard gene targeting methods in embryonic stem cells, using the previously described $129 / \mathrm{SvJ}$ targeting arms of MrgprB4 $4^{7}$. The lengths of $5^{\prime}$ and $3^{\prime}$ arms were 4.3 and $3.0 \mathrm{~kb}$, respectively. In one construct, the entire open reading frame of MrgprB4 (encoded by a single exon) was replaced with an mtdTomato-2A-NLSCre targeting cassette. This cassette was generated as a single open reading frame using overlapping PCR that connected the membrane-tagged tdTomato (containing the 8 amino acids of the MARCKS sequence (MGCCFSKT) fused to the N-terminus of the full length tdTomato, including its N-terminal methionine ${ }^{31}$ ) to a Nuclear Localization Signal (NLS)tagged Cre-recombinase via an intervening F2A sequence ${ }^{32}$. This cassette was ligated as a SacII/Sall fragment to the frt-PGK-neo-frt cassette ${ }^{33}$. It was then ligated in-frame to an AscI site at the endogenous ATG start codon of the MrgprB4 coding sequence. To generate $M r g p r B 4-E G F P f-2 A-F L P-A C N$ mice, the open reading frame of $M r g p r B 4$ was replaced by the EGFPf-2A-FLP cassette, where EGFPf (farnesylated EGFP; Clontech) was fused via the 2FA sequence to FLPo (codon optimized FLP recombinase ${ }^{34}$ ). This cassette was ligated to the self-excising loxP-flanked pol-II promoter-neomycin resistance cassette $\left(\mathrm{ACN}^{35}\right)$.

Homologous recombination was performed in mouse CJ7 embryonic stem (ES) cells following standard procedures. Correctly targeted ES clones were identified by PCR genotyping of genomic DNA isolated from G418-resistant clones using primer sets flanking the $5^{\prime}$ and $3^{\prime}$ arms of the targeting construct and were further confirmed by Southern Blot hybridization using probes that flanked the $5^{\prime}$ and $3^{\prime}$ arms of the targeting construct, as well as an internal probe to exclude illegitimate recombination events. Chimeric MrgprB4mtdTomato-2A-NLSCre-frt-PGK-neo-frt and MrgprB4-EGFPf-2A-FLP-ACN mice were produced by blastocyst injection of positive ES cells, and heterozygous progeny were 
generated by mating the chimeric mice to $\mathrm{C} 57 \mathrm{~B} 1 / 6 \mathrm{~N}$ mice. Back crossing to C57BL/6N mice was done for five or more generations.

\section{Neonatal mouse viral injections}

P1-P2 pups were removed from their cage and briefly submerged in an ice water bath inside a latex glove with their head up, until they appear anesthetized (3-5 min). The adequacy of anaesthesia was determined by toe pinch. Pups were then held gently by the head, with padding, the skin of the lower abdomen cleaned with an alcohol swab, and the animals were then immobilized in a plastic gel pocket with their ventral side up. A syringe (insulin syringe, $0.3 \mathrm{~cm}^{3}, 8 \mathrm{~mm}$ length, $31 \mathrm{G}$ needle) was used to inject AAV8 virus $(20-25 \mu \mathrm{l}$ containing $10^{10} \mathrm{AAV} 8$ particles, titered by dot blot hybridization or by genome copy number (using quantitative real time PCR, qPCR) intraperitoneally (i.p.), avoiding any visible milk spot. The pups were then covered with nesting material and placed on a water circulating heating pad until they began moving. Following this recovery period they were returned to their dam and observed for the appearance of a milk spot, indicating that they were healthy and suckling.

\section{Virus production}

AAV8 virus particles were produced using crude Iodixanol purification as described in ${ }^{36}$ and concentrated using a Millipore Ultra-15 unit (\#UFC910024).

\section{Immunofluorescence}

Adult mice (8-16 weeks old) were anesthetized with ketamine/xylazine and perfused with 20 $\mathrm{ml} 0.1 \mathrm{M}$ phosphate buffer solution (PBS; $\mathrm{pH} 7.4 ; 4^{\circ} \mathrm{C}$ ) followed by $25 \mathrm{ml} 4 \%$ paraformaldehyde (PFA) in PBS $\left(4^{\circ} \mathrm{C}\right)$. Dorsal root ganglia (DRG) were dissected from the perfused mice, postfixed in $4 \%$ PFA at $4^{\circ} \mathrm{C}$ for $5 \mathrm{~min}$, cryoprotected in $20 \%$ sucrose in PBS at $4^{\circ} \mathrm{C}$ for $24 \mathrm{hr}$, and frozen in OCT at $-80^{\circ} \mathrm{C}$. Tissues were sectioned at $20 \mu \mathrm{m}$ with a cryostat. The sections collected on slides were dried at $37^{\circ} \mathrm{C}$ for $15 \mathrm{~min}$. The slides were washed with PBS containing $0.2 \%$ Triton X-100 (PBT) and blocked with $10 \%$ goat/donkey serum in PBT for $30 \mathrm{~min}$. All sections were incubated overnight with primary antibodies diluted in blocking solution at $4^{\circ} \mathrm{C}$. The primary antibodies used were: rabbit anti-GFP (A-11122; Molecular Probes; 1:1000), rabbit anti-hrGFP (240142; Stratagene;1:200) and chicken anti-GFP (GFP1020;Aves Labs;1:1000). Following incubation with primary antibody, sections were washed with PBT and incubated with secondary antibodies at room temperature for $2 \mathrm{hr}$. Secondary antibodies were diluted 1:250 in blocking solution and were conjugated to Alexa-488 or Alexa 568 (Molecular Probes). Sections were counterstained with TO-PRO-3 (Molecular Probes), washed with PBT and mounted with Vectashield. Images were obtained using an Oympus Confocal Microscope system.

\section{Electrophysiological Recording in Ex Vivo Skin-Nerve preparations}

The ex-vivo somatosensory system preparation has been described in detail previously 26 . Briefly, adult MrgprB4-EGFP-2A-FLP mice were anesthetized with a mixture of ketamine $(90 \mathrm{mg} / \mathrm{kg})$ and xylozine $(10 \mathrm{mg} / \mathrm{kg})$, the skin of the dorsal hindpaw and limb was shaved and then the mice were transcardially perfused with chilled and oxygenated artificial cerebral spinal fluid. Surgical dissection was performed to isolate intact the hemisected spinal cord, L2-L3 dorsal roots and DRGs, saphenous nerves and innervated skin from the left or right hindlimbs. The skin was pinned hairy side up on an elevated platform, keeping the dermal side perfused and the epidermis dry. Bath temperature was maintained at $31^{\circ} \mathrm{C}$. $\mathrm{EGFP}^{+}$cells were targeted using fluorescent microscopy and DIC optics. Recording electrodes containing $5 \%$ Neurobiotin (NB) in $1 \mathrm{M}$ potassium acetate. A small amount of $<1 \%$ lucifer yellow was added to the solution for better visualization of the microelectrode tip under fluorescent 
illumination. Following impalement of a targeted neuron projecting through the saphenous nerve we first searched for its receptive field by stroking the skin using a fine camel-hair brush. Next the skin was searched using a small glass rod. We next applied thermal stimuli by flooding the skin surface with first cold $\left(0^{\circ} \mathrm{C}\right)$ and then hot $\left(52^{\circ} \mathrm{C}\right)$ buffered saline. Finally, in some of the experiments the skin was then treated with a cocktail of inflammatory compounds $(10 \mu \mathrm{M}$ histamine, $10 \mu \mathrm{M}$ bradykinin, $10 \mu \mathrm{M}$ serotonin, and $10 \mu \mathrm{M}$ prostaglandin $\mathrm{E}_{2}$, in 50\% DMSO and 50\% buffered Krebs solution at pH6), for 3-5 minutes to determine if the cells were either chemosensory or whether they could be sensitized to respond to the other stimulus modalities.

Summary of electrophysiology results-We recorded from $25 \mathrm{EGFP}^{+}$positive cells from 10 saphenous nerve preparations made from MrgprB4-EGFP-2A-FLP mice. None of these 25 cells could be activated with mechanical stimulation of the skin. Of these 21 were also thoroughly tested for thermal sensitivity and were found to be unresponsive. Finally 4 cells were tested with mechanical thermal and chemical stimuli (inflammatory soup) and all 4 remained unresponsive.

\section{Calcium Imaging}

Mice two months or older were sedated by I.P. injection of a mix of Ketamine $(100 \mathrm{mg} / \mathrm{kg})$, Xylazine $(15 \mathrm{mg} / \mathrm{kg})$, Acepromazine $(2.5 \mathrm{mg} / \mathrm{kg}$ in $0.9 \% \mathrm{NaCl})$. During imaging, body temperature was maintained at $37^{\circ} \mathrm{C}$ with a heating blanket.

A dorsal laminectomy was performed mostly at spinal level L2-L4 (but occasionally at L1L3) as described ${ }^{30}$ but without removing the dura. The spinal column was stabilized using Narishige STS-A spinal clamps ${ }^{29}$. In addition we used a head holding adaptor from Kopf (923-B Mouse Gas Anesthesia Head Holder) that has installed an anesthesia/gas mask for positioning the mouse head. In this apparatus the gas is applied through a standard hose barb positioned above the nose on the mask. The inlet fills a large gas chamber around the snout, a second hose barb below the mask is provided for vacuuming off excess, expelled gasses. The animals were maintained under continuous anesthesia for the duration of the imaging experiments with 1-2\% isofluorane or with hourly injections of the above ketamine mix. A well was built around the exposed spinal cord using Gelseal (Amersham Biosciences Corp) and Kwik Sil Adhesive (WPI). Warm imaging solution (in mM: $130 \mathrm{NaCl}, 3 \mathrm{KCl}, 2.5$ $\mathrm{CaCl} 2,0.6$ 6.H20.MgCl2, 10 Hepes w/o Na, $1.2 \mathrm{NaHC03,10}$ Glucose, $\mathrm{Ph} 7.45$ with $\mathrm{NaOH}$ ) $\left(37^{\circ} \mathrm{C}\right)$ was repeatedly applied to prevent drying and maintain tissue integrity, and to allow the use of immersion objectives. During imaging the body temperature of the animals was maintained at $37^{\circ} \mathrm{C}$ with a heating blanket and an air -therm heater (WPI) placed inside the microscope area.

Imaging experiments were performed under a 2-photon laser scanning microscope (Ultima, Prairie Instruments Inc.). Live images were acquired at 8-12 frames per second, at depths below the pia ranging from 100-250 $\mu \mathrm{m}$, using an Olympus 40X 0.8 N.A water immersion objective, at $128 \times 128$ pixel resolution with a laser tuned to $940 \mathrm{~nm}$ wavelength, and emission filters $525 / 50 \mathrm{~nm}$ and $595 / 50 \mathrm{~nm}$ for green and red fluorescence respectively. Laser power was adjusted to be $20-25 \mathrm{~mW}$ at the focal plane (maximally $35 \mathrm{~mW}$ ), depending on the imaging depth and level of expression of GCaMP3.0. Focal planes containing fibers activated by stimulation of a given peripheral area were identified by trial and error. tdTomato fluorescence was used to identify MrgprB4 ${ }^{+}$fibers until photobleaching occurred.

\section{Stimulus delivery during imaging experiments}

Mechanical stimuli-Brushing stimuli were delivered using a sable paint brush No. 5 . Pinching stimuli were delivered using serrated forceps (Adson-Graefe tissue forceps, Fine 
Science Tools, Cat\#11030-12). A touch sensor was designed to allow detection of a finger touch to a conductive band (copper) mounted on the paint brush (Supplementary Materials and Methods Fig. 1a, b). The function of the sensor amplifier/brush is to allow the coordinated movement of the brush tip with a light touch of the sensor band to produce a TTL (+5VDC) compatible voltage pulse that can be time-stamped to the image acquisition. For the pinching stimulation the touch sensor was modified to detect closure of the forceps. This adjustment was accomplished by mounting a plastic screw on the forceps so that the contact closure occurred at a consistent position (Supplementary Materials and Methods Fig. $1 b)$.

The circuitry inside the touch sensor box was designed as follows (see circuit diagram in Supplementary Materials and Methods Fig. 1c): the stimulus device (brush or forceps) was attached by a small wire with a male pin at the end. A 2 meter cable with a matching female receptacle and a BNC connector conveyed the electrical signal to the touch sensor amplifier. The probe input on the amplifier was connected to $+2.5 \mathrm{VDC}$ through a $10 \mathrm{M} \Omega$ resistor. This point was attached to a high impedance follower. The shield (outer part of the coaxial cable) on the probe wire is "driven" by being connected to the output of the follower. This provides a low impedance shield to keep electrical interference from coupling to the touch probe input line. When a touch is made, the output of the follower amplifier has a noise envelope (primarily 60/120 Hz) picked up by the body of the person touching the probe band (or the metal body of the forceps). The signal from the follower amplifier is rectified and injected into the positive input of a voltage comparator. The minus input of this circuit is connected to the wiper of a potentiometer on the front panel that provides a sensitivity adjustment. This adjustment allows for the wide range of touch sensitivity that is needed. When the voltage on the plus input (signal from the probe amplifier) exceeds the voltage on the minus input (set by the potentiometer) the output of the comparator is increased. The output of this comparator is conveyed to a BNC connector on the panel as a TTL pulse. The voltage level on this BNC remains high $(+5 \mathrm{~V})$ as long as the "touch" is being made. The signal is internally directed to a three position switch that allows for an LED to be lit or a tone to be generated, enabling visual or auditory confirmation of times when stimulation is performed. The TTL pulse is recorded by the Trigger Sync program (Prairie) which is time locked with the two photon image acquisition system (Prairie View, Prairie), thereby identifying imaging frames at which the mechanical stimuli were applied.

We concurrently recorded where on the animal the mechanical stimulation was applied. First, a dim red grid was projected onto the mouse (so as to have the least interference with the detection of the green fluorescence) using a laser pico projector (MicroVision, SHOWWX) to deliminate a coordinate system for stimulation. Then the movement of the brush and the location in the peripheral areas of the brushes were recorded using a camera (Basler, A601f-2).

Delivery of chemical stimuli to the spinal cord- $\mathrm{KCl}$, final concentration $\left(60 \mathrm{mM}^{37}\right)$ a, $\beta$-methylATP $\left(5 \mathrm{mM}^{38}\right)$ and $\mathrm{CNO}(1.5 \mathrm{mM})$ were delivered manually to the imaging bath using a pipetman.

Delivery of chemical stimuli to the periphery- $a, \beta$-methylATP $(10 \mu 1$ from $1 \mathrm{mM}$ solution) and capsaicin [10 $\mu \mathrm{l}$ from $1 \mathrm{mM}$ solution (10\% DMSO in saline)] was injected in the ventral and dorsal hindpaw of $\mathrm{MrgD}$ and $\mathrm{MrgB} 4$ mice respectively, using a syringe pump (WPI, Inc., sp200i syringe pump). The timing of the injection was controlled by the two photon image acquisition system and associated software (Prairie View and Trigger Sync, Prairie Technologies) to link it with image acquisition. 


\section{Analysis of Imaging Data}

GCaMP3.0 responses were quantified using custom software written in Matlab (VivoViewer software). Initially, the raw data were filtered by smoothing using a Gaussian filter. The filter is represented by a $3 \times 3$ matrix with values proportional to a 2-D Gaussian with its peak at the center, $\mathrm{SD}=0.5$, and normalized so that the matrix's entries sum to 1 . The filtered value for each pixel $=$ (its original value $\times$ the filter value in the center $)+($ original values of the adjacent pixels each multiplied by their corresponding filter values). To calculate values for pixels at the edge of the image, the image is treated as though there are pixels beyond the edge with values equal to those of the nearest edge pixel. Next, the images were subjected to background subtraction to remove excess background noise. This was accomplished by drawing an ROI around a region without any visible structures and calculating the average pixel value in that background ROI, for each frame used for analysis. This value was then subtracted from every pixel in the corresponding frame.

The average fluorescence intensity, $\mathrm{F}_{\mathrm{av}}$, was measured by calculating the average (background-subtracted) pixel values in a given region-of-interest (ROI), for each image frame recorded during a time interval spanning before and during the stimulation period. The $\mathrm{F}_{\mathrm{av}}$ was then converted to $\Delta \mathrm{F} / \mathrm{F}$ using the formula $\Delta \mathrm{F} / \mathrm{F}=\left(\mathrm{F}_{\mathrm{av}}-\mathrm{F}_{0}\right) / \mathrm{F}_{0}$, where $\mathrm{F}_{0}$ is the baseline fluorescence value, measured as the average pixel intensity during the first 2-11 frames of each imaging experiment. The resulting time series of $\Delta \mathrm{F} / \mathrm{F}$ in a given ROI was smoothed using a moving average with a window of 3 frames. For a window of size $M$ the following equation is used:

For a time series, $\mathrm{f}$, of $\mathrm{N}$ frames and a window size of $\mathrm{M}$ for the moving average (where $\mathrm{M}$ is an odd integer), the nth term of the new time series, F, is given by

$$
F_{n}=\sum_{i=n-m}^{n+m} \frac{f_{i}}{2 m+1}
$$

where $m=\frac{M-1}{2}$ if both

$$
n>\frac{M-1}{2} \text { and } n \leq N-\frac{M-1}{2} \text { are true. }
$$

other wise,

$$
\operatorname{m=min}\{n-1, N-n\}
$$

For the calculation of the trial average curves (e.g Fig 2e, g) for mechanical stimuli we used a 7 -frame smoothing window. Sections of the $\Delta \mathrm{F} / \mathrm{F}$ time series during which a stimulus occurred were collected for multiple trials, aligned to the onset of the stimulus, and averaged to find the mean response curve. Since repeated mechanical stimuli were delivered during each experimental trial, in order to be consistent each $\Delta \mathrm{F} / \mathrm{F}$ trace was calculated for a period of 5 frames just prior to each stimulus onset and for the subsequent 20 or 40 frames (i.e. the first frame of these 20-40 frame series coincided with the initiation of the stimulus). From these values we calculated the mean peak $\Delta \mathrm{F} / \mathrm{F}$ (MPI $\Delta \mathrm{F} / \mathrm{F}_{\text {peak }}$ ) and area under the curve for all the applied stimuli across trials. The average $\Delta \mathrm{F} / \mathrm{Fs}$ for specific ROIs in the same field of view were tested for statistical significance by repeated measures ANOVA, followed by Bonferoni's-corrected post hoc comparison of means. 
In the case of chemical stimulation (delivered either to the spinal cord or to the periphery) typically a single trial was performed for a given mouse, due to the difficulty of maintaining the same focal plane during the period of application of the chemical to the spinal cord or the period required for diffusion of the liquid bolus delivered for peripheral injection, respectively. In these cases, therefore, the MPI $\Delta \mathrm{F} / \mathrm{F}_{\text {peak }}$ before and during the stimulation period were calculated for multiple mice imaged using ROIs of similar size, and were compared for statistical significance (relative to pre-stimulus baseline) by repeated measures ANOVA, followed by Bonferoni's-corrected post hoc comparison of means (unless stated otherwise).

The $\Delta \mathrm{F} / \mathrm{F}$ values in supplementary Fig. $7 \mathbf{b}$ and $\mathbf{e}$ were corrected for photobleaching as described ${ }^{39}$.

\section{Behaviour}

The conditioned place preference (CPP) protocol was based on previous studies ${ }^{13,17}$. As we hypothesized a positive valence effect of activation of MrgB4 neurons, we used a biased compartment assignment procedure, in which activation of the neurons is tested for its ability to increase the time spent in the initially non-preferred chamber ${ }^{20}$. The CPP apparatus consisted of a rectangular chamber divided into three compartments (300x150x150 mm per compartment), connected via an opening (50x50 mm) in each delimiting wall. The two side (test) compartments were designed to have different visual and tactile cues, by having distinct walls (horizontal or vertical alternating white and black stripes) and distinct floors (different shapes of floor grids with big or small square holes). In addition a 1 inch-diameter polyvinylchloride (PVC) pipe coupler (two schedule 40 wall thickness), either threaded or smooth, was placed in the center of each side compartment to enrich for tactile cues ${ }^{17}$. The center compartment was a neutral plastic enclosure (cf. Figure 4a). This design was chosen so as to promote a compartment preference assignment for each mouse. A video tracking system (Noldus Ethovision) recorded all animal movements.

Since our hypothesis is based on the social reward mediated by social contact in juvenile mice ${ }^{17}$ the mice used were approximately one month old. Following weaning the mice were maintained in social groups and left undisturbed until the start of the CPP assay. The paradigm was completed in 6 days. The day prior to pre-testing the mice were socially isolated in their home cage. On Day 1 of the procedure each mouse was placed in the central compartment and allowed to freely explore the entire apparatus for $30 \mathrm{~min}$ (pre-test).

Following the pre-test the initial preference of each mouse for a given side compartment was recorded. With our apparatus design most of the mice showed an initial preference for one of the two side compartments. Conditioning was initiated on Day 2 and encompassed 4 sessions performed on 4 consecutive days. In the first conditioning session mice were injected i.p with CNO $(5 \mathrm{mg} / \mathrm{kg})^{19}$ (or saline of an equivalent volume for some control mice) and placed for 1 hour (based on the observation that CNO effects peak between 45 and 50 min after administration ${ }^{19}$ ) in the initially non-preferred (I.N.P.) compartment. On Day 3, during the second conditioning session, all mice were injected with saline and confined for 1 hour in the opposite (i.e., initially preferred, I.P) compartment. (The second conditioning session was performed the following day since $\mathrm{CNO}$ effects last for 9 hours ${ }^{19}$.) On Day 4 and Day 5 the first and second conditioning sessions were repeated, respectively. The time between the ip injections of $\mathrm{CNO}$ or saline and the placement of the mice in the compartment was between 5-10 min, which is compatible with the time that is needed for CNO to start having an effect ${ }^{19}$. On Day 6 , the mice were tested for their side compartment preference by placing them in the center compartment and allowing them to freely explore the entire apparatus for $30 \mathrm{~min}$ (post test). All sessions were conducted blind to the genotype/injected virus of each mouse. For the Conditioned Place Aversion (CPA assay) the 
mice remained group housed until the day before the pre-test. Following the pre-test, on the first day of the conditioning session the mice were injected with saline and confined in the I.N.P compartment. The second day of conditioning the mice were injected with CNO (except for the saline control mice) and placed in the I.P compartment. On the $3^{\text {rd }}$ and $4^{\text {th }}$ day of conditioning the $1^{\text {st }}$ and $2^{\text {nd }}$ sessions of conditioning were repeated, respectively. On the $6^{\text {th }}$ day the mice were tested for their preference in the three compartment arena.

\section{Behavioral Data Analysis}

Difference scores for each chamber (time in chamber during post-test minus time in chamber during pre-test) were analyzed for statistical significance (significant difference from zero) using simple or repeated one way ANOVA $(\mathrm{p}<0.05)$ followed by a Bonferronicorrected post hoc comparison of means. Statistical analysis of all other metrics was performed using a repeated two way mixed model ANOVA (unless otherwise stated) (with each group as the between-subject variable and pre-training vs. post-training as the withinsubject variable). Detection of a significant interaction and/or main effect was followed by Bonferroni-corrected post hoc comparison of means.

\section{Drugs}

Clozapine-N-oxide (CNO) was obtained from Biomol International, and dissolved in saline.

\section{Supplementary Material}

Refer to Web version on PubMed Central for supplementary material.

\section{Acknowledgments}

We thank Robert Robertson for programming and imaging data analysis, Mike Walsh and Tim Heitzman for the stimulus delivery system and associated electronics, Moriel Zelikowsky for help with statistical analysis of behavioral data, Hidehiko Inagaki for experimental advice and helpful comments on the manuscript, Shirley Pease for help with generation of knock-in mice, Natalie Verduzco, Kwan Lee and Reyna Sauza for mice colony maintenance, Meike Visel and John Flannery (UC Berkeley) for training in AAV8 preparation, Aileen Anderson and Chelsea Pagan (UCI) for initial experiments using their stereotaxic apparatus, Jie Zhang and Allan Basbaum (UCSF) for teaching the dorsal laminectomy, D. Davalos and Katerina Akassoglou (UCSF) and Helge Johanssen (BRI, Zurich) for help with the in vivo imaging preparation, Leon Lagnado and Benjamin Odermatt for SypHy and SyGCamp2 plasmids, Catie Shea and Monica Martinez for technical assistance, Holly Oates-Barker for lab management and Gina Mancuso for administrative assistance. This work was supported by NIH grants 5PO1NS-48499 and 5R01 NS023476, and by fellowships from EMBO and the Human Frontiers Science Program (S.V.) and the Helen Hay Whitney Foundation (A.W.). D.J.A. is an Investigator of the Howard Hughes Medical Institute.

\section{REFERENCES}

1. Morrison I, Loken LS, Olausson H. The skin as a social organ. Exp Brain Res. 2010; 204:305-314. doi:10.1007/s00221-009-2007-y. [PubMed: 19771420]

2. Olausson H, Wessberg J, Morrison I, McGlone F, Vallbo A. The neurophysiology of unmyelinated tactile afferents. Neurosci Biobehav Rev. 2010; 34:185-191. doi:10.1016/j.neubiorev.2008.09.011. [PubMed: 18952123]

3. Dunbar RI. The social role of touch in humans and primates: behavioural function and neurobiological mechanisms. Neurosci Biobehav Rev. 2010; 34:260-268. doi:10.1016/j.neubiorev. 2008.07.001. [PubMed: 18662717]

4. McGlone F, Vallbo AB, Olausson H, Loken L, Wessberg J. Discriminative touch and emotional touch. Canadian Journal of Experimental Psychology/Revue canadienne de psychologie expérimentale. 2007; 61:173-183. doi:10.1037/cjep2007019.

5. Dong X, Han S, Zylka MJ, Simon MI, Anderson DJ. A diverse family of GPCRs expressed in specific subsets of nociceptive sensory neurons. Cell. 2001; 106:619-632. [PubMed: 11551509] 
6. Zylka MJ, Dong X, Southwell AL, Anderson DJ. Atypical expansion in mice of the sensory neuronspecific Mrg G protein-coupled receptor family. Proc Natl Acad Sci U S A. 2003; 100:1004310048. [PubMed: 12909716]

7. Liu Q, et al. Molecular genetic visualization of a rare subset of unmyelinated sensory neurons that may detect gentle touch. Nat Neurosci. 2007; 10:946-948. doi:10.1038/nn1937. [PubMed: 17618277]

8. Wessberg J, Olausson H, Fernström KW, Vallbo AB. Receptive field properties of unmyelinated tactile afferents in the human skin. Journal of neurophysiology. 2003; 89:1567-1575. doi:10.1152/ jn.00256.2002. [PubMed: 12626628]

9. Li L, et al. The functional organization of cutaneous low-threshold mechanosensory neurons. Cell. 2011; 147:1615-1627. doi:10.1016/j.cell.2011.11.027. [PubMed: 22196735]

10. Rau KK, et al. Mrgprd enhances excitability in specific populations of cutaneous murine polymodal nociceptors. J Neurosci. 2009; 29:8612-8619. doi:10.1523/JNEUROSCI. 1057-09.2009. [PubMed: 19571152]

11. Zylka MJ, Rice FL, Anderson DJ. Topographically distinct epidermal nociceptive circuits revealed by axonal tracers targeted to Mrgprd. Neuron. 2005; 45:17-25. doi:10.1016/j.neuron.2004.12.015. [PubMed: 15629699]

12. Cavanaugh DJ, et al. Distinct subsets of unmyelinated primary sensory fibers mediate behavioral responses to noxious thermal and mechanical stimuli. Proc Natl Acad Sci U S A. 2009; 106:90759080. doi:10.1073/pnas.0901507106. [PubMed: 19451647]

13. Tzschentke TM. Measuring reward with the conditioned place preference (CPP) paradigm: update of the last decade. Addict Biol. 2007; 12:227-462. doi:10.1111/j.1369-1600.2007.00070.x. [PubMed: 17678505]

14. Tian L, et al. Imaging neural activity in worms, flies and mice with improved GCaMP calcium indicators. Nat Methods. 2009; 6:875-881. doi:10.1038/nmeth.1398. [PubMed: 19898485]

15. Dussor G, Zylka MJ, Anderson DJ, McCleskey EW. Cutaneous sensory neurons expressing the Mrgprd receptor sense extracellular ATP and are putative nociceptors. J Neurophysiol. 2008; 99:1581-1589. doi:10.1152/jn.01396.2007. [PubMed: 18234974]

16. Edin BB, Essick GK, Trulsson M, Olsson KA. Receptor encoding of moving tactile stimuli in humans. I. Temporal pattern of discharge of individual low-threshold mechanoreceptors. J Neurosci. 1995; 15:830-847. [PubMed: 7823184]

17. Panksepp JB, Lahvis GP. Social reward among juvenile mice. Genes Brain Behav. 2007; 6:661671. doi:10.1111/j.1601-183X.2006.00295.x. [PubMed: 17212648]

18. Panksepp J. Cross-species affective neuroscience decoding of the primal affective experiences of humans and related animals. PLoS One. 2011; 6:e21236. doi:10.1371/journal.pone.0021236 PONE-D-11-02008 [pii]. [PubMed: 21915252]

19. Alexander GM, et al. Remote control of neuronal activity in transgenic mice expressing evolved G protein-coupled receptors. Neuron. 2009; 63:27-39. doi:10.1016/j.neuron.2009.06.014. [PubMed: 19607790]

20. Le Foll B, Goldberg SR. Nicotine induces conditioned place preferences over a large range of doses in rats. Psychopharmacology (Berl). 2005; 178:481-492. doi:10.1007/s00213-004-2021-5. [PubMed: 15765262]

21. Cunningham CL, Ferree NK, Howard MA. Apparatus bias and place conditioning with ethanol in mice. Psychopharmacology (Berl). 2003; 170:409-422. doi:10.1007/s00213-003-1559-y. [PubMed: 12955296]

22. Johansen JP, Fields HL, Manning BH. The affective component of pain in rodents: direct evidence for a contribution of the anterior cingulate cortex. Proc Natl Acad Sci U S A. 2001; 98:8077-8082. doi:10.1073/pnas.141218998 141218998 [pii]. [PubMed: 11416168]

23. LaBuda CJ, Fuchs PN. A behavioral test paradigm to measure the aversive quality of inflammatory and neuropathic pain in rats. Exp Neurol. 2000; 163:490-494. doi:10.1006/exnr.2000.7395 S0014-4886(00)97395-3 [pii]. [PubMed: 10833324]

24. Shields SD, Cavanaugh DJ, Lee H, Anderson DJ, Basbaum AI. Pain behavior in the formalin test persists after ablation of the great majority of C-fiber nociceptors. Pain. 2010; 151:422-429. doi:S0304-3959(10)00468-9 [pii] 10.1016/j.pain.2010.08.001. [PubMed: 20832171] 
25. Loken LS, Wessberg J, Morrison I, McGlone F, Olausson H. Coding of pleasant touch by unmyelinated afferents in humans. Nat Neurosci. 2009; 12:547-548. doi:10.1038/nn.2312. [PubMed: 19363489]

26. Woodbury CJ, Ritter AM, Koerber HR. Central anatomy of individual rapidly adapting lowthreshold mechanoreceptors innervating the "hairy" skin of newborn mice: early maturation of hair follicle afferents. J Comp Neurol. 2001; 436:304-323. [PubMed: 11438932]

27. Olausson $\mathrm{H}$, et al. Unmyelinated tactile afferents signal touch and project to insular cortex. Nature neuroscience. 2002; 5:900-904. doi:10.1038/nn896.

28. Foust KD, Poirier A, Pacak CA, Mandel RJ, Flotte TR. Neonatal intraperitoneal or intravenous injections of recombinant adeno-associated virus type 8 transduce dorsal root ganglia and lower motor neurons. Hum Gene Ther. 2008; 19:61-70. doi:10.1089/hum.2007.093. [PubMed: 18052722]

29. Davalos D, et al. Stable in vivo imaging of densely populated glia, axons and blood vessels in the mouse spinal cord using two-photon microscopy. J Neurosci Methods. 2008; 169:1-7. doi: 10.1016/j.jneumeth.2007.11.011. [PubMed: 18192022]

30. Johannssen HC, Helmchen F. In vivo Ca2+ imaging of dorsal horn neuronal populations in mouse spinal cord. J Physiol. 2010; 588:3397-3402. doi:10.1113/jphysiol.2010.191833. [PubMed: 20660563]

31. Muzumdar MD, Tasic B, Miyamichi K, Li L, Luo L. A global double-fluorescent Cre reporter mouse. Genesis. 2007; 45:593-605. doi:10.1002/dvg.20335. [PubMed: 17868096]

32. de Felipe P, et al. E unum pluribus: multiple proteins from a self-processing polyprotein. Trends Biotechnol. 2006; 24:68-75. doi:10.1016/j.tibtech.2005.12.006. [PubMed: 16380176]

33. Meyers EN, Lewandoski M, Martin GR. An Fgf8 mutant allelic series generated by Cre- and Flpmediated recombination. Nat Genet. 1998; 18:136-141. doi:10.1038/ng0298-136. [PubMed: 9462741]

34. Raymond CS, Soriano P. ROSA26Flpo deleter mice promote efficient inversion of conditional gene traps in vivo. Genesis. 2010; 48:603-606. doi:10.1002/dvg.20659. [PubMed: 20665730]

35. Bunting M, Bernstein KE, Greer JM, Capecchi MR, Thomas KR. Targeting genes for self-excision in the germ line. Genes Dev. 1999; 13:1524-1528. [PubMed: 10385621]

36. Zolotukhin $\mathrm{S}$, et al. Recombinant adeno-associated virus purification using novel methods improves infectious titer and yield. Gene Ther. 1999; 6:973-985. doi:10.1038/sj.gt.3300938. [PubMed: 10455399]

37. Hamada FN, et al. An internal thermal sensor controlling temperature preference in Drosophila. Nature. 2008; 454:217-220. doi:10.1038/nature07001. [PubMed: 18548007]

38. Hamilton SG, McMahon SB, Lewin GR. Selective activation of nociceptors by P2X receptor agonists in normal and inflamed rat skin. J Physiol. 2001; 534:437-445. [PubMed: 11454962]

39. Berry JA, Cervantes-Sandoval I, Nicholas EP, Davis RL. Dopamine Is Required for Learning and Forgetting in Drosophila. Neuron. 2012; 74:530-542. doi:10.1016/j.neuron.2012.04.007. [PubMed: 22578504] 


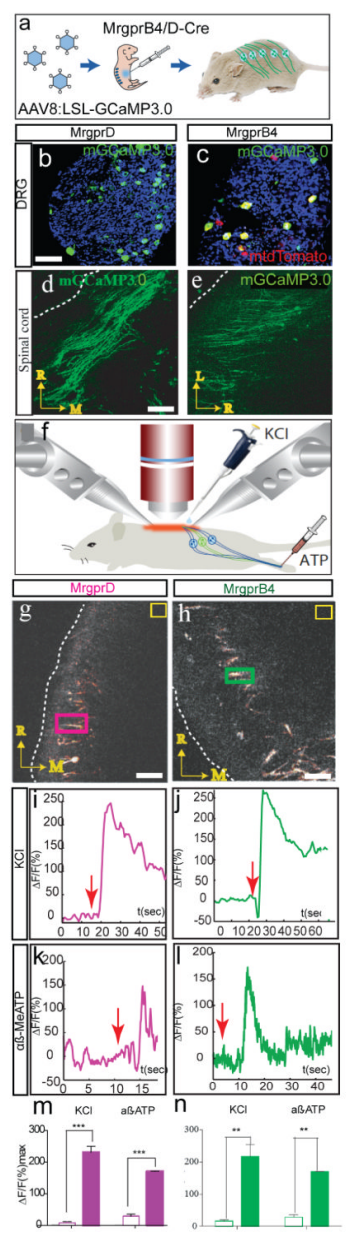

Figure 1. In vivo calcium imaging in genetically defined subsets of primary sensory neurons a, Schematic illustrating AAV infection. LSL, loxP-STOP-loxP cassette. b-e, mGCaMP3.0 expression in somata $(\mathbf{b}, \mathbf{c})$ and central afferent fibers $(\mathbf{d}, \mathbf{e})$ of $\mathrm{MrgprD}^{+}(\mathbf{b}, \mathbf{d})$ or $\mathrm{MrgprB}^{+}$ (c, e) neurons in adult mice. Dashed lines indicate lateral margin of spinal cord. Scale bars in $(\mathbf{b}, \mathbf{d})=50$ and $45 \mu \mathrm{m}$, respectively. f, schematic illustrating imaging preparation; components not to scale. g-n, Calcium transients in the central projections of $\mathrm{MrgprD}^{+}(\mathbf{g}, \mathbf{i}$, $\mathbf{k , ~ m})$ or MrgprB4${ }^{+}(\mathbf{h}, \mathbf{j}, \mathbf{l}, \mathbf{n})$ neurons, evoked by direct application of $\mathrm{KCl}$ to the spinal cord $(\mathbf{i}, \mathbf{j})$ or (in a different animal) peripheral injection of $a, \beta$-methylene $\operatorname{ATP}(\mathbf{k}, \mathbf{l})$. Colored rectangles in $(\mathbf{g}, \mathbf{h})$ indicate Regions-Of-Interest (ROIs) used in (i, j), respectively; yellow boxes are regions for background subtraction. Scale bar in $(\mathbf{g}, \mathbf{h})=40$ and $20 \mu \mathrm{m}$, respectively. Red arrows (i-l) indicate time of stimulus delivery. (m, n) Quantification of peak $\Delta \mathrm{F} / \mathrm{F}$ values before (open bars) vs. after (filled bars) stimulation. $* *, \mathrm{p}<.01 ; * * *, \mathrm{p}<$. 001. All data in this and other figures are mean \pm SEM. 

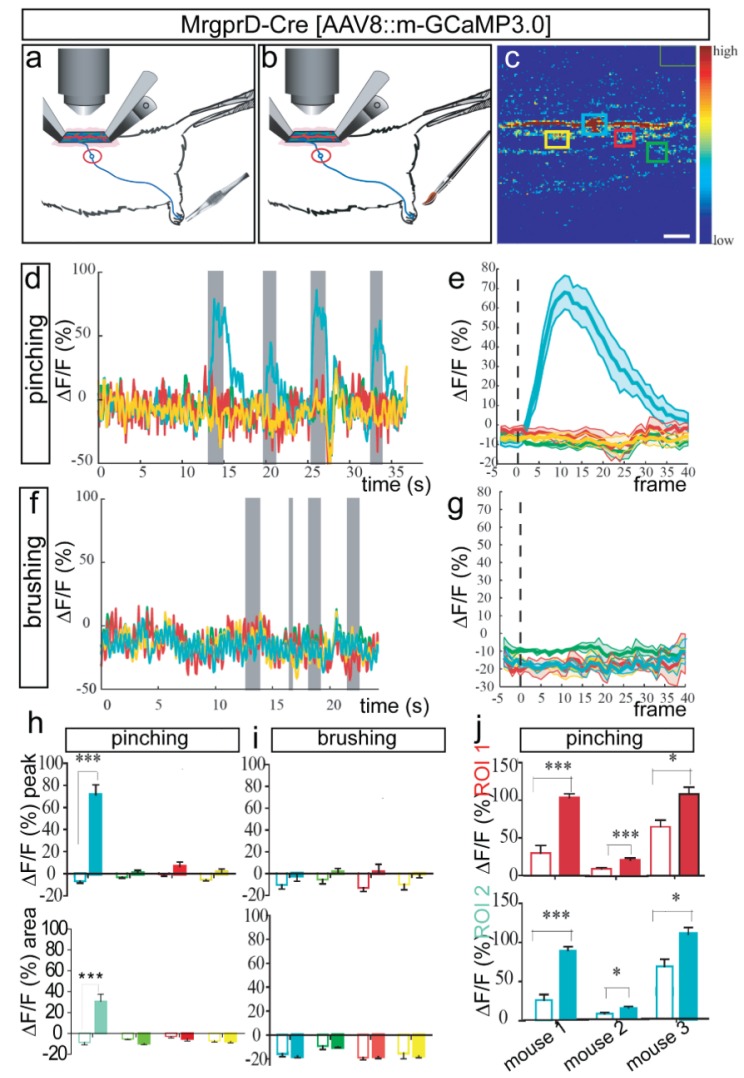

Figure 2. Activation of MrgprD fibers by pinching

a, b, Schematics illustrating pinching (a) and stroking (b) stimuli. c, ROIs used for imaging in (d-i). Green rectangle (upper right) is region for background subtraction. Scale bar, $9 \mu \mathrm{m}$. d, Superimposed traces from different color-coded ROIs (c) in a single trial consisting of 4 pinch stimuli (gray bars). e, Average response to pinching in a single animal ( $\mathrm{n}=4$ trials, 7 stimuli total). See also Supplementary Fig. 5a-e. f, Response to 4 brushing stimuli (gray bars) delivered to pinch-sensitive digit (d), in same ROI (c). See also Supplementary Fig. 9g-1. g, Average response to brushing ( $\mathrm{n}=1$ mouse/2 trials, 7 stimuli). h, i, MPI $\Delta \mathrm{F} / \mathrm{F}_{\text {peak }}$ (upper) or integrated area (lower) from curves in (e, g), respectively. Open and filled bars are 5 frames before and 40 frames after stimulus delivery, respectively (see Supplementary Note 9). $\mathbf{j}$, MPI $\Delta F / F_{\text {peak }}$ in two different ROIs (red and turquoise bars) from each of three mice. See also Supplementary Table 2. 


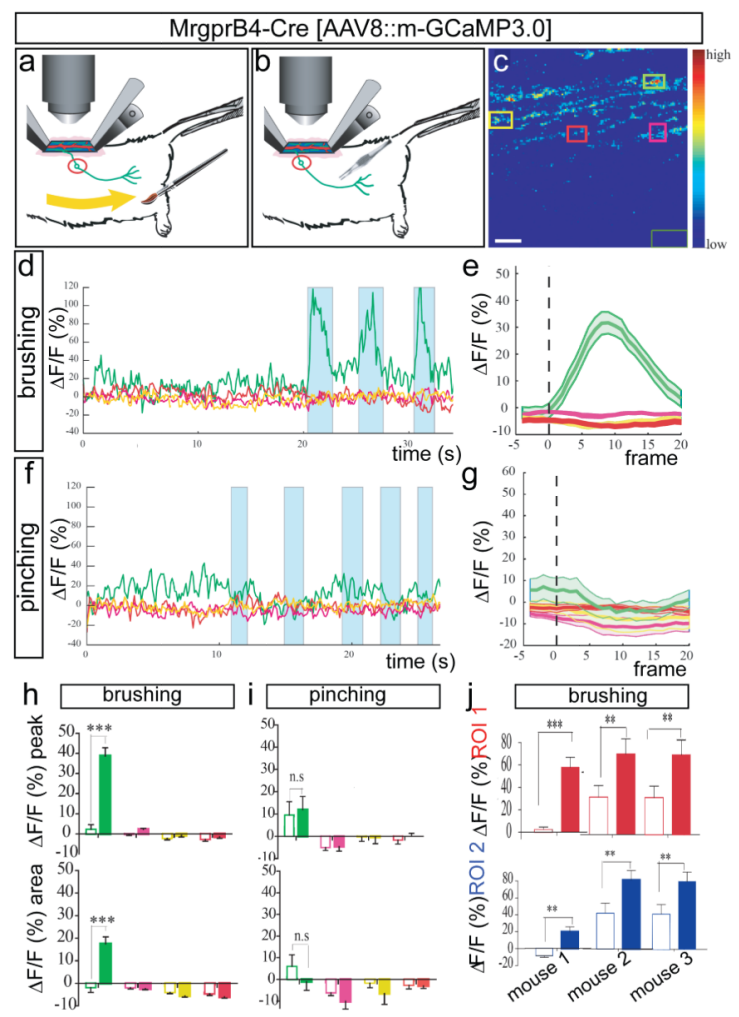

Figure 3. Activation of MRGPRB4 fibres by stroking

a, b, Schematics illustrating brushing (a) and pinching (b) stimuli. c, F image of GCaMP3.0 at one frame point during stimulation and ROIs used for imaging in $\mathbf{d}-\mathbf{i}$. The dark-green rectangle (lower right) is the region used for background subtraction. Scale bar, $8.5 \mu \mathrm{m}$. d, Superimposed traces from different colour coded ROIs (c) in a single trial of three brush stimuli (turquoise bars). e, Average response to brushing from a single mouse ( $n=5$ trials, , 3-6 stimuli per trial). See also Supplementary Fig. $5 \mathrm{~g}-\mathrm{j}$. f, Response to five pinching stimuli (turquoise bars) in brush-sensitive region (d), in same ROI (c). See also

Supplementary Fig. 9a-f. g, Average response to pinching from the same animal ( $\mathrm{n}=2$ trials, 10 stimuli total). $\mathrm{h}, \mathrm{i}, \mathrm{MPI} \Delta \mathrm{F} / \mathrm{F}_{\text {peak }}$ (upper) or integrated area (lower) calculated from the curves in e, $\mathbf{g}$, respectively. Open and filled bars are 5 frames before and 20 frames after stimulus delivery, respectively. NS, not significant. $\mathbf{j}, \mathrm{MPI} \Delta \mathrm{F} / \mathrm{F}_{\text {peak }}$ in two different ROIs (red and blue graphs) from each of three independent mice. See also Supplementary Table 3. $* * \mathrm{P}<0.01 ; * * * \mathrm{P}<0.001$. All data are mean $+/-$ s.e.m. 


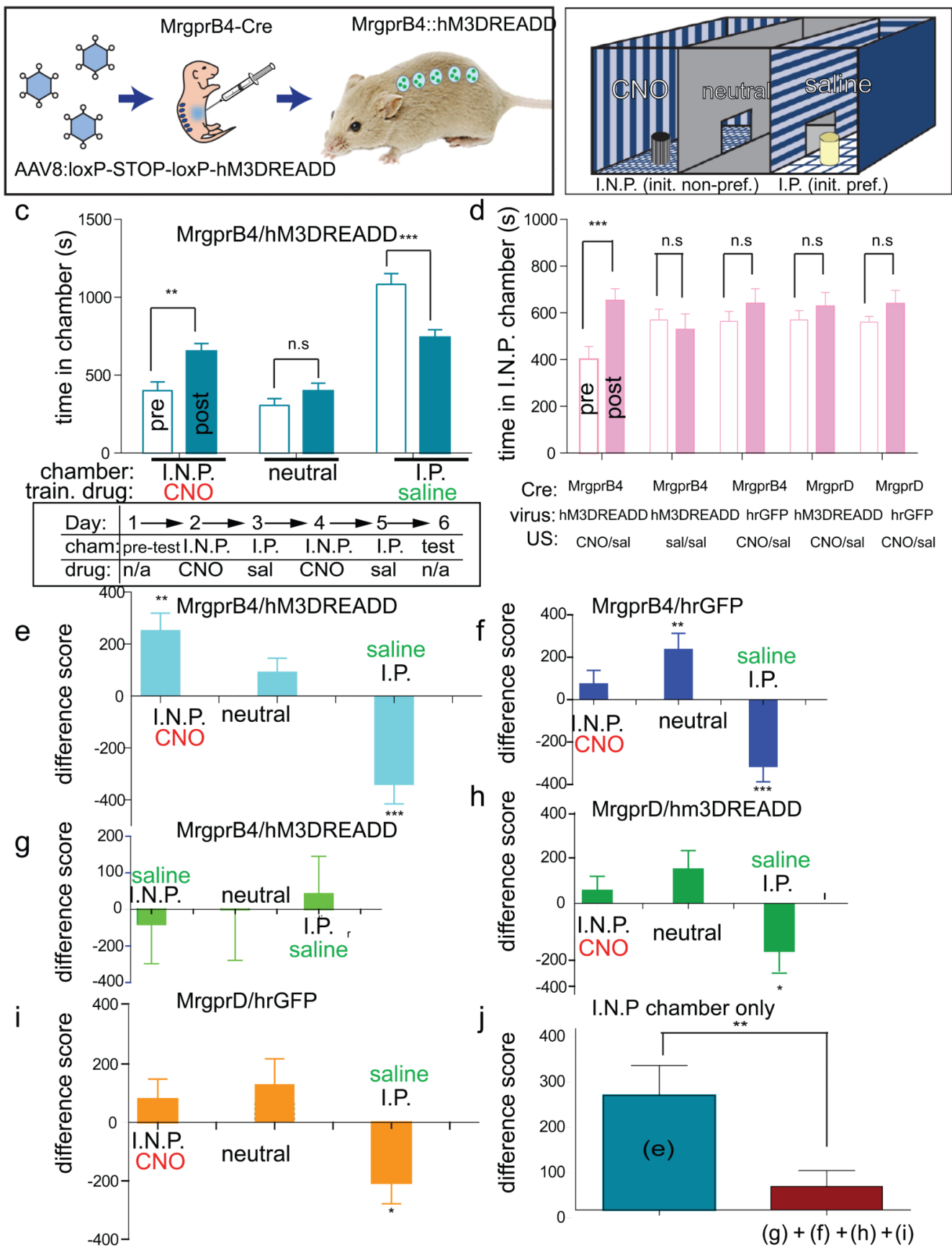

Figure 4. Activation of MRGPRB4 neurons promotes conditioned place preference $\mathbf{a}, \mathbf{b}$, Schematic of experiment (a) and CPP apparatus (b). I.N.P. and I.P. indicate initially non-preferred and preferred chambers, respectively (c, schematic, 'pre-test'). c, Top: absolute time (s) in each chamber before (open bars; 'pre') versus after (filled bars; 'post') conditioning for the experimental group. Train drug indicates $\mathrm{CNO}$ or saline paired with the indicated chamber. Bottom: schematic of experimental design. Cham., chamber. d, Time in I.N.P. chamber for experimental (replotted from panel $\mathbf{c}$ for direct comparison) and control groups. ${ }^{* *} \mathrm{P}<0.01 ; * * * \mathrm{P}<0.001$; NS, not significant. See Supplementary Note 10 for F values and Supplementary Fig. 13. e-i, Difference scores ((time in indicated chamber after 
training) - (time in chamber before training) ) for experimental $(\mathbf{e}, \mathrm{n}=15)$ and control $(\mathbf{f}, \mathbf{g}, \mathrm{h}$, $\mathrm{i}, \mathrm{n}=9,6,8,10$, respectively) groups. $\mathbf{j}$, Comparison of mean difference scores for the I.N.P. chamber for the experimental (e) and the pooled control (f, $\mathbf{g}, \mathrm{h}, \mathrm{i})$ groups. There was no significant difference between control groups. $*<\mathrm{P}, 0.05, * * \mathrm{P}<0.01 ; * * * \mathrm{P}<0.001$. 